Heute gehört es fast schon zum guten Ton, Invalide als Profiteure zu beschuldigen. Und nun befinden wir Ärztinnen und Ärzte uns plötzlich im gleichen Boot: «Ärzte lügen oder sind unfähig, wenn sie IV-Berichte ausfüllen, und müssen deshalb verurteilt werden!» Das ist die Be- deutung einer demnächst im Parlament eingebrachten Motion, zu der Dr. med. Gerhard Ebner und unser Jurist Hanspeter Kuhn Stellung nehmen. Vielen Dank für diese wichtige Klarstellung!

Dr. med. Jacques de Haller, Präsident der FMH

\title{
Motion Hutter: Haftung für Ärzte bei Beihilfe zur Scheininvalidität
}

Die Motion [1] behauptet: «Während früher der Patient nur im Ausnahmefall als krank angesehen wurde, wird heute so lange gesucht, bis eine zum Patient passende Krankheit gefunden wird. Ärzte definieren den Krankheitsbegriff zusammen mit den Versicherten täglich neu - mit dem Resultat, dass die Invalidenversicherung (...) Milliardensummen für Fälle ausgibt, denen kein wirklich invaliditätsrelevanter Gesundheitsschaden zugrunde liegt.» Die Motion will deshalb das IVG ergänzen, so «dass Ärzte künftig für Arztzeugnisse haftbar gemacht werden können, wenn sich diese als objektiv unhaltbar herausstellen und dadurch der Invalidenversicherung Kosten entstanden sind.»

Aus Sicht der FMH ist diese Behauptung undifferenziert, die Schlussfolgerungen sind falsch und die vorgeschlagene Lösung unnötig.

\section{Schon heute verfassen Ärzte \\ ihre Zeugnisse und Gutachten nicht im rechtsfreien Raum}

Über die Gründe, weshalb die IV-Renten bis vor einigen Jahren zunahmen, wurde schon viel geschrieben: so über das Herausfallen, um nicht zu sagen Abschieben, von Menschen mit eingeschränkter Leistungsfähigkeit bei zunehmendem Produktivitätsdruck unter Mithilfe aller Beteiligten und ungenügender Kontrolle durch die IV. Die Rechtssprechung (Kriterien zur Beurteilung der Zumutbarkeit), die Ärzte (Entwicklung von Leitlinien) und die Gesetzgebung (4. und 5. IVG-Revision) haben hierauf reagiert, die Zahl der Neuberentungen nimmt seit 2004 ab [2].

Eine aktuelle Studie des BSV [3] widerlegt die Behauptung, dass falsche Diagnosen von immer neuen Krankheiten zu IV-Berentungen führen. Sie zeigt auch auf, dass ärztliche Abklärungen umfassend und differenziert sind; teilweise ungenügend ist die Darstellung der Funktionsstörungen, welche die Arbeitsfähigkeit transparent belegen sollten. Für deren Be- urteilung gibt es bis heute - zumindest für psychische Störungen - keine evidenzbasierten Kriterien, an denen sich Ärzte, Leitlinienverantwortliche und Rechtsanwender orientieren könnten [4]. Entscheidend für die Qualität von Berichten wie Gutachten sind klare und akzeptierte Standards. Eine Projektgruppe im Auftrag des BSV entwickelt derzeit formale wie inhaltliche Kriterien für psychiatrische IV-Begutachtungen; die formalen Kriterien werden auf Ende Jahr fertiggestellt und anschliessend evaluiert. Die Rechtssprechung erhält mit diesen Kriterien Grundlagen für möglichst objektive Urteile.

Schon heute verfassen Ärzte ihre Zeugnisse und Gutachten nicht im rechtsfreien Raum: «Ärztliche Zeugnisse, Berichte und Gutachten sind Urkunden. Bei deren Ausstellung haben Arzt und Ärztin alle Sorgfalt anzuwenden, um nach bestem Wissen ihre ärztliche Überzeugung auszudrücken» (FMH-Standesordnung). 2008 hat das Bundesgericht zwei Ärzte verurteilt, weil sie Gefälligkeitszeugnisse ausgestellt hatten.

Eine verschärfte Haftpflicht, wie sie die Motion fordert, würde einzig dazu führen, dass sich die Ärztinnen und Ärzte aus Angst vor Haftpflichtprozessen in der Beurteilung nicht mehr festlegen würden - mit der Folge, dass Berichte und Gutachten für die Rechtssprechung unbrauchbar würden.

Dr. med. Gerhard Ebner M.H.A, Mitglied der Projektgruppe und Leiter einer Arbeitsgruppe zur Entwicklung von Leitlinien zur psychiatrischen IV-Begutachtung

Hanspeter Kuhn, Fürsprecher, stv. Generalsekretär FMH

\section{Literatur}

1 Motion Jasmin Hutter 07.3685.

2 Pressemitteilung BSV, 14.9.2006.

3 Baer N, Frick U, Fasel T. Dossieranalyse der Invalidisierungen aus psychischen Gründen. 2009; www.bsv.admin.ch

4 Dittmann V, Ebner G, Herdt J, Junge C, Träbert S. Literaturstudie als Grundlage zur Entwicklung von evidenzbasierten Gütekriterien zur Beurteilung von psychischen Behinderungen. 2009; www.bsv.admin.ch 